\title{
Alcohol consumption and bone mineral density in elderly women
}

\author{
Isolde Sommer ${ }^{1, *}+$, Arja T Erkkilä ${ }^{1}$, Ritva Järvinen ${ }^{1}$, Jaakko Mursu' ${ }^{1}$, Joonas Sirola ${ }^{2,3}$, \\ Jukka S Jurvelin ${ }^{4,5}$, Heikki Kröger ${ }^{2,3}$ and Marjo Tuppurainen ${ }^{2,6}$ \\ ${ }^{1}$ Institute of Public Health and Clinical Nutrition, University of Eastern Finland, Kuopio, Finland: ${ }^{2}$ Bone and \\ Cartilage Research Unit, Clinical Research Center, University of Eastern Finland, Kuopio, Finland: ${ }^{3}$ Departments \\ of Orthopedics and Traumatology and Handsurgery, Kuopio University Hospital, Kuopio, Finland: ${ }^{4}$ Department \\ of Clinical Physiology, Nuclear Medicine, Kuopio University Hospital, Kuopio, Finland: ${ }^{5}$ Department of Applied \\ Physics, University of Eastern Finland, Kuopio, Finland: ${ }^{6}$ Department of Obstetrics and Gynecology, Kuopio \\ University Hospital, Kuopio, Finland
}

Submitted 31 January 2012: Final revision received 17 May 2012: Accepted 1 June 2012: First published online 17 July 2012

\begin{abstract}
Objective: Findings regarding alcohol consumption and bone mineral density (BMD) in elderly women have been inconsistent. The objective of the present study was to explore the association of alcohol intake with BMD in elderly women.

Design: This cohort study included women from the population-based Kuopio Osteoporosis Risk Factor and Prevention - Fracture Prevention Study (OSTPREFPS). Alcohol intake and potential confounders were assessed at baseline and after 3 years of follow-up using a lifestyle questionnaire. In addition, an FFQ was distributed in the third year to measure dietary intake, including alcohol. Women underwent BMD measurements at the femoral neck and lumbar spine at baseline and after 3 years of follow-up.

Setting: Kuopio Province, Finland.

Subjects: Three hundred elderly women (mean age $67 \cdot 8$ years) who provided both BMD measurements and FFQ data.

Results: Alcohol consumption estimated from the FFQ and lifestyle questionnaire was significantly associated with BMD at both measurement sites after adjustment for potential confounders, including lifestyle and dietary factors $(P<0 \cdot 05)$. Using the FFQ, women drinking $>3$ alcoholic drinks/week had significantly higher BMD than abstainers, $12 \cdot 0 \%$ at the femoral neck and $9 \cdot 2 \%$ at the lumbar spine. Results based on the lifestyle questionnaire showed higher BMD values for all alcohol-consuming women at the femoral neck and for women drinking 1-3 alcoholic beverages/week at the lumbar spine, compared with non-users. Conclusions: The results from OSTPRE-FPS suggest that low to moderate alcohol intake may exert protective effects on bone health in elderly women.
\end{abstract}

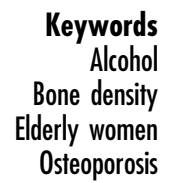

eywords

one density

Osteoporosis
Osteoporosis and related fractures have major consequences for the health of elderly women worldwide. Low bone mineral density (BMD) has been shown to be a strong predictor of an enhanced fracture risk $^{(1,2)}$. In addition to unmodifiable risk factors such as age and sex, several nutritional and lifestyle factors including low $\mathrm{Ca}$ intake and low physical activity are recognized as important risk factors for the development of osteoporosis ${ }^{(3)}$. Alcohol intake as a potential contributing factor to osteoporosis

$\dagger$ Correspondence address: School of Health Sciences and Social Work, University of Portsmouth, James Watson West, 2 King Richard 1st Road, Portsmouth PO1 2FR, UK. and osteoporotic fractures was first described in $1965^{(4)}$. Since then, the negative impact of chronic heavy alcohol consumption on bone health has been recognized ${ }^{(5)}$. Long-term alcohol use has been shown to exert direct and indirect effects on bone turnover and remodelling, leading to decreased BMD and increased risk of fractures ${ }^{(6)}$. Alcohol may directly worsen bone health by impairing proliferation and function of osteoblasts ${ }^{(7)}$, causing malabsorption, increased renal excretion and disruption of Ca-regulating hormones such as parathyroid hormone, calcitonin and vitamin D metabolites. Also, insufficient dietary intake related to alcohol may indirectly interfere with bone homeostasis ${ }^{(8,9)}$. In contrast to these harmful effects of 
alcohol abuse, several studies have reported that moderate alcohol use may decrease fracture rates and increase bone density, mostly in postmenopausal women ${ }^{(10-23)}$. However, others showed no significant association between alcohol intake and $\mathrm{BMD}^{(24-28)}$, and one reported a negative relationship between BMD at the lumbar spine and lifetime beer consumption $^{(29)}$.

As with CVD, the relationship between alcohol intake and bone health may be U-shaped ${ }^{(30)}$. Moderate alcohol consumption has been suggested to increase oestrogen levels in postmenopausal women and subsequently prevent the development of osteoporosis. Studies, especially more recent ones ${ }^{(31,32)}$, have suggested that moderate alcohol intake may be associated with increased oestrogen levels in postmenopausal women. However, the findings regarding the effect of alcohol intake on BMD in elderly women are inconclusive and more studies with extensive adjustments for potential confounders are needed. To continue this line of investigation, the present study examined the association between alcohol intake and BMD at the lumbar spine and proximal femur in a subsample of the Kuopio Osteoporosis Risk Factor and Prevention Study (OSTPRE) population.

\section{Materials and methods}

\section{Study design and participants}

The present study was based on the Kuopio Osteoporosis Risk Factor and Prevention - Fracture Prevention Study (OSTPRE-FPS) which began in 2003 in Kuopio, Finland ${ }^{(33)}$. The primary aim of the study was to determine whether vitamin D and Ca supplementation would prevent bone loss in postmenopausal women in a non-placebo-controlled, parallel-group trial. The ethics committee of Kuopio University Hospital approved the OSTPRE-FPS in October 2001. The trial was registered at Clinictrials.gov under identifier NCT00592917 (registration date: 2 January 2008). Written informed consent was obtained from the study participants at baseline enquiry. The OSTPRE-FPS population ( $n$ 5407) was selected from the population-based OSTPRE cohort of 13100 peri- and postmenopausal women born in 1932-1941 ${ }^{(34)}$. The inclusion criteria for OSTPREFPS were: being a minimum of 65 years of age at the end of November 2002; living in Kuopio Province at the onset of the trial; and not having been included in the OSTPRE sample in which BMD measurements were conducted. Willingness to participate in a vitamin D and Ca supplementation trial was enquired via mail. A response rate of $63.5 \%$ led to a study population of 3432 women, which was randomized into two groups of equal size. From this study population, a subsample of 750 women was randomly selected to take part in a clinical trial. Of these, a total of 606 participants started the trial.

The baseline measurements took place between February 2003 and May 2004 and the follow-up measurements between January 2006 and May 2007 (mean follow-up time $2 \cdot 8$ (SD $0 \cdot 4$ ) years). In both examinations, lifestyle, health status and use of drugs were assessed using questionnaires, and BMD measurements were undertaken. The supplementation group received daily cholecalciferol $20 \mu \mathrm{g}(800 \mathrm{IU})+\mathrm{Ca} 1000 \mathrm{mg}$ via prescription, while the control group received neither supplementation nor placebo. A total of 593 participants completed the study. Of them, 544 women underwent BMD measurements at the femoral neck and 480 women at the lumbar spine. Additionally, an FFQ was distributed at the follow-up examination. However, not all women were willing to complete the FFQ. This left a total of 341 women who underwent both baseline and 3-year BMD measurements and returned the FFQ. Thereof, fifteen women were excluded because of implausible energy intake $(>14644 \mathrm{~kJ}(>3500 \mathrm{kcal}))$ and twenty-six were excluded due to incomplete data, leaving 300 women to be included in the final analysis (Fig. 1).

\section{Questionnaires}

The OSTPRE-FPS questionnaire (lifestyle questionnaire) was used to collect information on lifestyle, health status and medications at baseline and alcohol consumption at baseline and follow-up. Although it has not been validated to measure alcohol consumption, it was successfully used to demonstrate the association between alcohol intake and spinal BMD in an earlier study ${ }^{(3)}$. In the lifestyle questionnaire, participants were asked questions about age, current and past hormone therapy (HT), current smoking status, mobility, diseases and medications, and use of non-prescribed $\mathrm{Ca}$ and/or vitamin D supplements. Non-prescribed Ca and/or vitamin D supplements included those available over the counter, which were consumed in addition to the prescribed $\mathrm{Ca}$ and vitamin D intervention of the trial. The mean duration of HT use in years ( 10.9 years) was substituted for fifty participants who reported that they had used HT but did not remember its exact duration. Criteria for restricted mobility were: (i) not able to walk more than $1 \mathrm{~km}$; (ii) not able to walk more than $100 \mathrm{~m}$; (iii) only able to move indoors; and (iv) immobile. To assess alcohol consumption, participants were asked to quantify their intake of beer/cider (bottles), wine (glasses) or spirits/strong alcohol (portions) during the last 4 weeks. A 'drink' referred to a bottle of beer/cider, a glass of wine or one portion of spirits/strong alcohol. In Finland, the average portion size for a bottle of beer/cider is $330 \mathrm{ml}$, for a glass of wine is $120 \mathrm{ml}$ and for a shot of spirits/strong alcohol $40 \mathrm{ml}$.

The FFQ assessed the usual dietary intake at follow-up. Participants were asked to report their usual consumption of beer/cider (1 bottle $=330 \mathrm{ml})$, red or white wine $(120 \mathrm{ml})$ or spirits $(40 \mathrm{ml})$ by selecting one of nine frequency categories: 'never or very seldom', '1-3 times per month', 'once per week', '2-4 times per week', '5-6 times per week', 'once per day', '2-3 times per day', ‘4-5 times 


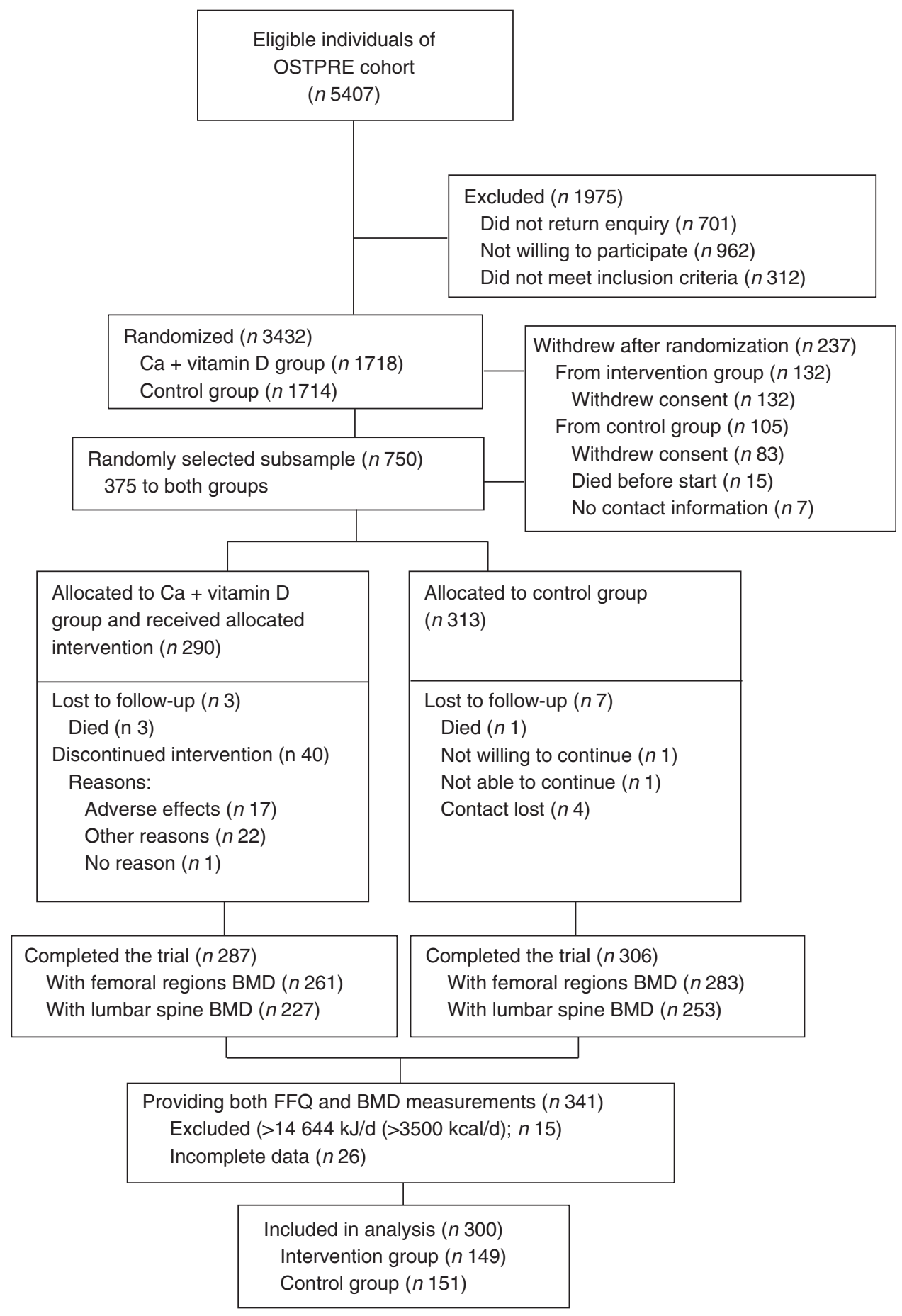

Fig. 1 Flowchart of the subsample of the Kuopio Osteoporosis Risk Factor and Prevention - Fracture Prevention Study (OSTPREFPS) trial (BMD, bone mineral density)

per day' or 'more than 5 times per day'. For analysis, average alcohol intakes per week were calculated. Total dietary intakes of energy, protein, K, Ca and vitamin D, out of all questioned food items, were computed. These are considered potential confounders and were therefore used as covariates in the statistical models. The FFQ was validated with food records for energy and nutrient intakes, and agreement between the two measurement tools was moderate ${ }^{(35)}$.

\section{Antbropometric measurements}

Weight of the participant was measured with a digital calibrated scale (Philips, type HF 351/00) and height with a calibrated wall meter during the baseline research visit. 
BMI $\left(\mathrm{kg} / \mathrm{m}^{2}\right)$ was computed by dividing the weight (in kilograms) by the square of height (in metres).

\section{Measurements of bone mineral density}

BMD measurements were performed at the lumbar spine (L2-L4) and left femoral neck with dual-energy X-ray absorptiometry (DXA; Lunar DPX, Madison, WI, USA) at baseline and year 3 examinations. The quality control of the DXA measurements was run daily. The in vitro long-term reproducibility (CV) for the total femur region was $0 \cdot 3 \%$. Measurements were carried out by following the guidelines of the instrument's manufacturer. The technical quality of each DXA measurement and analysis was carefully checked and those with measurement errors were not included in the statistical analyses ${ }^{(36)}$.

\section{Statistical analyses}

Average alcohol consumption derived from the lifestyle questionnaires or the FFQ was divided into four categories: no use; $<1$ drink/week; $1-3$ drinks/week; and $>3$ drinks/ week. These cut-offs produced categories with sufficient numbers of participants to be able to generate more stable estimates. Since the number of participants in the highest category was relatively small, more detailed analysis to detect potential differences between moderate $v$. heavy drinkers was not possible due to limited statistical power. Associations between alcohol intake and other variables potentially affecting BMD were analysed using one-way ANOVA for continuous variables and the $\chi^{2}$ test for categorical variables. These potential confounders were identified through literature review. Linear mixed-effects modelling was used to examine the relationship between alcohol consumption (added as the mean of two measurement time points using the lifestyle questionnaire, or added as one measurement time point at year 3 using the FFQ) and BMD changes over time (added as a time-varying covariate with two measurement time points). No significant changes in alcohol consumption were found and therefore alcohol was entered into the model as the mean of baseline and follow-up. The possibility of an interaction between alcohol intake and the effect of the vitamin D and $\mathrm{Ca}$ intervention (study group) on BMD was also tested using the likelihood ratio test, and showed no significance. Therefore, results are presented for all women.

Models were run unadjusted in the first step (accounting for study group only; Model 1); then run adjusted for confounding factors assessed at baseline including age (years), BMI $\left(\mathrm{kg} / \mathrm{m}^{2}\right)$, whether a current smoker (yes/no), HT use current or past (yes/no), duration of HT use (years), use of non-prescribed $\mathrm{Ca}$ and vitamin $\mathrm{D}$ supplement in addition to intervention (yes/no), study group (intervention/control), restricted mobility (yes/no), disease or medication decreasing BMD (yes/no; Model 2); and finally dietary factors including total energy intake $(\mathrm{kJ} / \mathrm{d})$, dietary protein intake $(\mathrm{g} / \mathrm{d})$, dietary $\mathrm{K}$ intake $(\mathrm{mg} / \mathrm{d})$, dietary Ca intake $(\mathrm{mg} / \mathrm{d})$ and dietary vitamin D $(\mu \mathrm{g} / \mathrm{d})$ were added to the list of covariates
(Model 3). The diseases potentially affecting bone metabolism included hyperthyroidism, disease of the parathyroid gland, chronic liver disease, chronic intestinal disease, coeliac disease, ventricle operation, chronic nephropathy, arthritis, osteoporosis and lactose intolerance. Medications affecting bone metabolism included loop-diuretics, insulin, anti-epileptics, glucocorticoids and cancer chemotherapy. Results are shown as estimated marginal means. Analyses were conducted separately for alcohol consumption from the lifestyle questionnaire and for alcohol consumption from the FFQ. Statistical analyses were carried out with the statistical software packages SPSS for Windows version $15 \cdot 0$ (SPSS Inc.) and STATA version 9.0 (StataCorp). The level of significance was set at $0 \cdot 05$.

\section{Results}

Generally, alcohol consumption among the elderly women was low. The majority of the women were non-drinkers (lifestyle questionnaire ( $n$ 118), 39.9\%; FFQ ( $n$ 169), $56.3 \%$ ) or consumed $<1$ drink/week (lifestyle questionnaire ( $n 103), 34 \cdot 8 \%$; FFQ ( $n 69), 23 \cdot 0 \%$ ). A total of $18 \cdot 9 \%$ of women based on the lifestyle questionnaire ( $n$ 56) and $11.0 \%$ of women based on the FFQ ( $n$ 33) reported intake of 1-3 drinks/week. Only a very few participants consumed $>3$ drinks/week (lifestyle questionnaire ( $n$ 19), 6.4\%; FFQ ( $n$ 29), 9.7\%).

Mean alcohol intake measured by the lifestyle questionnaire at baseline and follow-up was 0.82 drinks/week, which was slightly lower than the mean alcohol intake that was obtained from the FFQ (0.93 drinks/week) at the follow-up measurement (Table 1). The mean BMD at the lumbar spine was increased at follow-up $\left(1.097 \mathrm{~g} / \mathrm{cm}^{3}\right)$ compared with baseline $\left(1.087 \mathrm{~g} / \mathrm{cm}^{3}\right)$. About half of the women $(54.5 \%)$ currently used or had used HT at some time in their life. Only $4 \cdot 8 \%$ of the women were current smokers.

According to their alcohol consumption categories assessed through the lifestyle questionnaire at baseline and follow-up, women in the higher categories of alcohol intake were more likely to be smokers (Table 2).

Alcohol use was significantly associated with BMD (Tables 3 and 4). Using the FFQ, women in the highest category of alcohol intake had significantly higher BMD than non-consumers at both measurement sites (Table 3). At the femoral neck, the $P$ value for the unadjusted model was $0 \cdot 020$ (Model 1). The strongest association between alcohol intake and BMD was observed after adjustment for both lifestyle and dietary factors $(P=0 \cdot 014)$. In this model, women consuming $>3$ alcoholic drinks/week had $12 \cdot 0 \%$ higher BMD values than abstainers (Model 3). Similar results were found for the lumbar spine, where the association between alcohol intake and BMD was strongest in the unadjusted model $(P=0 \cdot 002$; Model 1$)$. After adjustment for lifestyle and dietary factors, women 
Table 1 Characteristics of the study participants: elderly Finnish women ( $n$ 300), Kuopio Osteoporosis Risk Factor and Prevention - Fracture Prevention Study (OSTPRE-FPS)

\begin{tabular}{|c|c|c|}
\hline Characteristic & Mean or \% & SD \\
\hline \multicolumn{3}{|l|}{ Baseline (February 2003-May 2004) } \\
\hline Age (years; $n$ 298) & $67 \cdot 8$ & $1 \cdot 8$ \\
\hline BMl $\left(\mathrm{kg} / \mathrm{m}^{2} ; n\right.$ 298) & $28 \cdot 4$ & $4 \cdot 3$ \\
\hline Current or past $\mathrm{HT}$ use $(\% ; n$ 279) & $54 \cdot 5$ & - \\
\hline Duration of HT use (years; $n$ 153) & $11 \cdot 3$ & $5 \cdot 9$ \\
\hline Non-prescribed use of Ca and vitamin D supplements $(\% ; n$ 298) & $13 \cdot 8$ & - \\
\hline Current smoking $(\% ; n$ 293) & $4 \cdot 8$ & - \\
\hline Restricted mobility (\%; $n$ 292) & $6 \cdot 2$ & - \\
\hline Disease or medication decreasing BMD (\%; $n$ 298) & $31 \cdot 8$ & - \\
\hline \multicolumn{3}{|l|}{ Follow-up (January 2006-May 2007) } \\
\hline Total energy intake $(\mathrm{kJ} / \mathrm{d} ; n$ 300) & 8979 & 2297 \\
\hline Total energy intake (kcal/d; $n$ 300) & 2146 & 549 \\
\hline Dietary protein intake (g/d; $n 300)$ & $90 \cdot 7$ & $27 \cdot 3$ \\
\hline Dietary Ca intake $(\mathrm{mg} / \mathrm{d} ; n$ 300) & 1324 & 502 \\
\hline Dietary vitamin D intake $(\mu \mathrm{g} / \mathrm{d} ; n 300)$ & $10 \cdot 0$ & $4 \cdot 8$ \\
\hline Dietary K intake (mg/d; $n$ 300) & 4777 & 1301 \\
\hline \multicolumn{3}{|l|}{ Total alcohol intake (drinks/week) } \\
\hline Lifestyle questionnaire (average of baseline and follow-up; $n$ 296) & $0 \cdot 82$ & $1 \cdot 30$ \\
\hline FFQ (follow-up; $n$ 300) & 0.93 & $1 \cdot 87$ \\
\hline \multicolumn{3}{|l|}{$\mathrm{BMD}\left(\mathrm{g} / \mathrm{cm}^{3}\right)$} \\
\hline \multicolumn{3}{|l|}{ Femoral neck } \\
\hline Baseline ( $n$ 292) & 0.866 & $0 \cdot 127$ \\
\hline Follow-up ( $n$ 291) & $0 \cdot 852$ & $0 \cdot 121$ \\
\hline \multicolumn{3}{|l|}{ Lumbar spine } \\
\hline Baseline ( $n$ 252) & $1 \cdot 087$ & $0 \cdot 184$ \\
\hline Follow-up ( $n$ 247) & $1 \cdot 097$ & $0 \cdot 181$ \\
\hline \multicolumn{3}{|l|}{ Study group (\%) } \\
\hline Intervention & $49 \cdot 7$ & - \\
\hline Control & $50 \cdot 3$ & - \\
\hline
\end{tabular}

$\mathrm{HT}$, hormone therapy; BMD, bone mineral density.

in the highest category of alcohol intake had $9 \cdot 2 \%$ higher BMD compared with non-users ( $P=0 \cdot 006$; Model 3$)$.

Using the lifestyle questionnaire to assess alcohol intake, significant associations between alcohol intake and BMD at both bone sites were found in unadjusted and adjusted models (Table 4). At the femoral neck, the $P$ value in the unadjusted model was 0.008 (Model 1 ). Women who consumed any amount of alcohol (from $<1$ to $>3$ drinks/week) had significantly higher BMD than women who abstained from alcohol after adjustment for lifestyle and dietary factors (Model 3). Women drinking $>3$ alcoholic drinks/week had $7 \cdot 7 \%$ higher BMD than non-drinkers after adjustment for both lifestyle and dietary factors $(P=0.025$; Model 3$)$. Analysis at the lumbar spine showed that women consuming 1-3 and $>3$ alcoholic drinks/week had significantly higher BMD than non-drinkers in the unadjusted model $(P=0.008$ and $P=0.039$, respectively; Model 1 ). After adjustment for lifestyle and dietary factors, only women drinking 1-3 alcoholic drinks/week had significantly higher BMD $(6 \cdot 4 \%)$ than abstainers $(P=0 \cdot 019$; Model 3).

\section{Discussion}

The findings of the present study suggest that low alcohol consumption may be beneficially related to bone health in elderly women. Depending on the alcohol assessment method, alcohol consumption of $>3$ drinks/week (FFQ) or 1-3 and $>3$ drinks/week (lifestyle questionnaire) was positively associated with BMD at the femoral neck and lumbar spine. All associations between alcohol use and BMD at both measurement sites remained significant after adjustment for lifestyle and dietary factors (Model 3). Depending on measurement site, dietary factors, including total energy intake and dietary intakes of protein, $\mathrm{Ca}$, vitamin $\mathrm{D}$ and $\mathrm{K}$, strengthened or attenuated the association between alcohol and BMD. The latter have been identified as the most evident dietary factors affecting BMD in the literature ${ }^{(37-41)}$ and were therefore added to the analysis. These findings suggest that low alcohol consumption has an independent effect on BMD. However, other dietary issues and many other factors contribute to bone health.

Although earlier observations suggested a potential protective effect of moderate alcohol consumption on BMD at the femoral neck and/or lumbar spine in postmenopausal women ${ }^{\text {(10-12,14-16,18,20-23) }}$, highest BMD values were mostly found in women who consumed at least one drink daily. Among a group of peri- and postmenopausal women, alcohol consumption (mean intake of consumers was $125 \mathrm{~g}$ /week) was a positive predictor for spine $\mathrm{BMD}^{(3)}$. The women participating in the present study consumed at most 1-3 drinks/week or $>3$ drinks/week, respectively, 


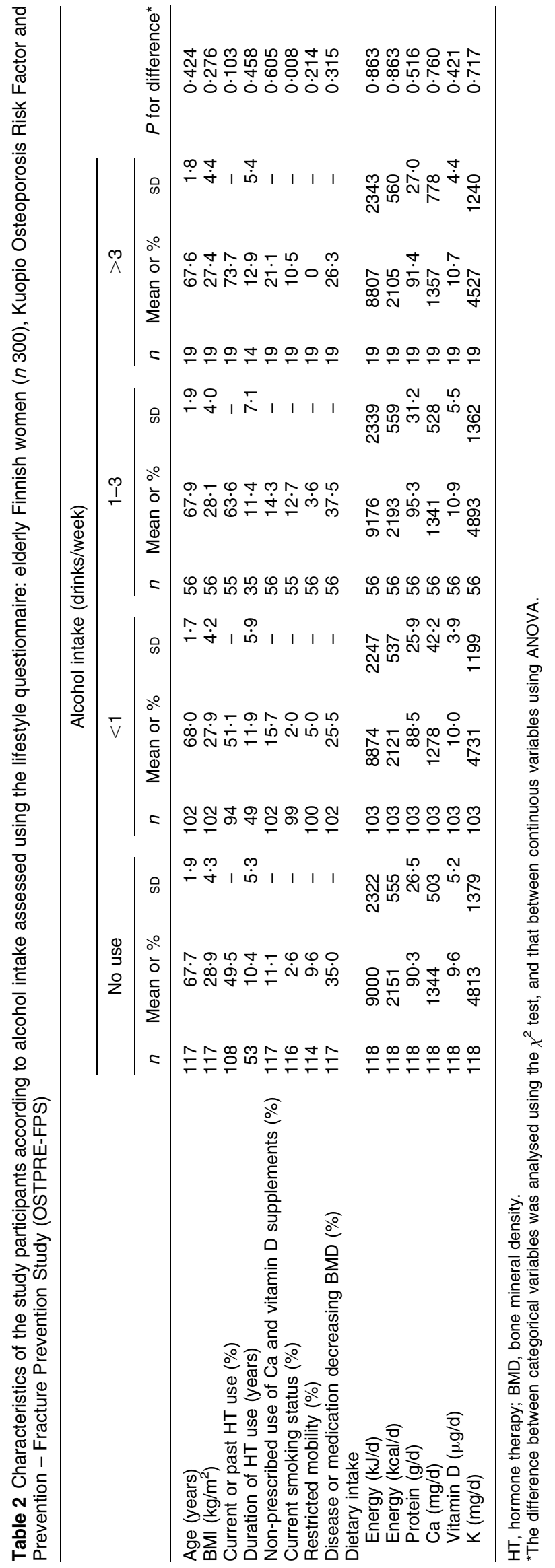

which corresponds to weekly alcohol intake of $10-30 \mathrm{~g}$ or more. Laitinen et al $^{(11)}$ and Rapuri et al $^{(18)}$ have previously reported a beneficial effect of low alcohol consumption on BMD. Laitinen et al. ${ }^{(11)}$ found higher BMD for postmenopausal women consuming 11-77g alcohol/week than for abstainers at all measurement sites (femoral neck, Ward's triangle, trochanter and lumbar spine). Similar results were found in the study conducted by Rapuri et al. ${ }^{(18)}$ where women with an alcohol consumption of $28 \cdot 7-57 \cdot 2 \mathrm{~g} /$ week had the highest lumbar spine, total body and mid-radius BMD compared with non-users. Therefore, alcohol consumption may already be beneficial for bone health at lower doses than previously assumed.

The average alcohol consumption of elderly women in the present study, 0.8-0.9 drinks/week, was relatively low, but comparable to previous estimates found for Finnish elderly ${ }^{(42)}$. Using an FFQ to measure alcohol intake, $37 \cdot 7 \%$ of women aged $65-69$ years reported to abstain from alcohol and $35 \cdot 3 \%$ reported to consume less than one alcoholic drink per week ${ }^{(42)}$. The present study found $39 \cdot 3 \%$ of elderly women to abstain from alcohol when using the lifestyle questionnaire and $56 \cdot 3 \%$ when using the FFQ data. Likewise, $34 \cdot 8 \%$ (lifestyle questionnaire) and $23.0 \%$ (FFQ) of elderly women consumed less than one alcoholic drink weekly in the present study. It has recently been reported ${ }^{(43)}$ that under-reporting of alcohol consumption most likely occurs when data are collected from an FFQ. Additionally, drinking behaviour and beliefs about alcohol differ between cultures and age groups. Studies assessing alcohol use among the elderly ( $>74$ years) in Finland found that alcohol use was less likely to be under-reported if it was in the context of medicinal use ${ }^{(44)}$. We cannot rule out the possibility that some women may have been misclassified and if so, the association between alcohol intake and BMD would be underestimated.

In line with the findings from the original OSTPRE-FPS trial $^{(33)}$ where BMD measurements at the lumbar spine had increased after 3 years irrespective of the intervention, results at the lumbar spine were also higher at the follow-up assessment in the present study. This observation has been made in earlier studies ${ }^{(45,46)}$ and could be explained by age-related degenerative changes, such as osteoarthritis, that have been shown to confound DXA measurements ${ }^{(47)}$. Since our analysis was adjusted for current and past HT use, duration of HT use and medications affecting BMD, we can rule out the potential impact of these on the BMD increase.

As reported in previous studies ${ }^{(12,14,18,19,21,25)}$ smoking and drinking habits tend to be strongly associated. Although the percentage of smokers in the current study population was low $(4 \cdot 8 \%)$, the proportion of smokers was higher among those consuming 1-3 or $>3$ alcoholic drinks/ week than among non-drinkers (12.7\% or $10 \cdot 5 \% v \cdot 2 \cdot 6 \%)$. Additionally, low body weight or low BMI, which are strong predictors of osteoporotic fractures ${ }^{(48)}$, frequently 
Table 3 Estimated marginal means derived from the linear mixed model predicting BMD change over time by category of alcohol intake assessed using the FFQ at follow-up: elderly Finnish women (n 300), Kuopio Osteoporosis Risk Factor and Prevention - Fracture Prevention Study (OSTPRE-FPS)

\begin{tabular}{|c|c|c|c|c|c|c|}
\hline & \multicolumn{2}{|c|}{ Model $1^{\star}$} & \multicolumn{2}{|c|}{ Model 2† } & \multicolumn{2}{|c|}{ Model 3‡ } \\
\hline & Mean & $95 \% \mathrm{Cl}$ & Mean & $95 \% \mathrm{Cl}$ & Mean & $95 \% \mathrm{Cl}$ \\
\hline \multicolumn{7}{|l|}{ Femoral neck } \\
\hline No use & 0.845 & $0 \cdot 827,0 \cdot 864$ & $0 \cdot 848$ & $0 \cdot 830,0.865$ & $0 \cdot 847$ & $0.829,0.865$ \\
\hline$<1$ drinks/week & 0.872 & $0.843,0.902$ & 0.875 & $0.848,0.902$ & 0.874 & $0.847,0.901$ \\
\hline 1-3 drinks/week & $0 \cdot 875$ & $0.833,0.917$ & $0 \cdot 879$ & $0.840,0.918$ & $0 \cdot 878$ & $0.840,0.917$ \\
\hline$>3$ drinks/week & $0.903 \S$ & $0.858,0.948$ & $0.899 \$$ & $0.858,0.941$ & $0.904 \S$ & $0.863,0.946$ \\
\hline \multicolumn{7}{|l|}{ Lumbar spine } \\
\hline No use & $1 \cdot 076$ & $1 \cdot 047,1 \cdot 105$ & $1 \cdot 079$ & $1 \cdot 051,1 \cdot 107$ & $1 \cdot 080$ & $1 \cdot 052,1 \cdot 108$ \\
\hline$<1$ drinks/week & 1.093 & $1 \cdot 047,1 \cdot 139$ & 1.094 & $1 \cdot 051,1 \cdot 137$ & 1.093 & $1 \cdot 050,1 \cdot 136$ \\
\hline 1-3 drinks/week & $1 \cdot 081$ & $1 \cdot 014,1 \cdot 148$ & $1 \cdot 089$ & $1 \cdot 026,1 \cdot 152$ & $1 \cdot 087$ & $1 \cdot 024,1 \cdot 150$ \\
\hline$>3$ drinks/week & $1 \cdot 192 \S$ & $1 \cdot 125,1 \cdot 261$ & $1 \cdot 183 \S$ & $1 \cdot 119,1 \cdot 247$ & $1 \cdot 179 \S$ & $1 \cdot 114,1 \cdot 243$ \\
\hline
\end{tabular}

BMD, bone mineral density; HT, hormone therapy.

*Adjusted for study group (intervention $v$. control).

tAdjusted for age, BMI, HT use, duration of HT use, current smoking, use of $\mathrm{Ca}$ and vitamin D supplements, restricted mobility, disease or medication decreasing $\mathrm{BMD}$, study group (intervention $v$. control).

$\ddagger$ Adjusted for age, BMI, HT use, duration of HT use, current smoking, use of $\mathrm{Ca}$ and vitamin D supplements, restricted mobility, disease or medication decreasing BMD, study group (intervention v. control), energy intake, dietary protein, dietary $\mathrm{Ca}$, dietary vitamin $\mathrm{D}$, dietary $\mathrm{K}$.

$\S$ The difference between category no use $v$. other categories is statistically significant at the 0.05 level.

Table 4 Estimated marginal means derived from the linear mixed model predicting BMD change over time by category of alcohol intake assessed using the lifestyle questionnaire at baseline and follow-up (mean): elderly Finnish women ( $n$ 300), Kuopio Osteoporosis Risk Factor and Prevention - Fracture Prevention Study (OSTPRE-FPS)

\begin{tabular}{|c|c|c|c|c|c|c|}
\hline & \multicolumn{2}{|c|}{ Model $1^{*}$} & \multicolumn{2}{|c|}{ Model 2† } & \multicolumn{2}{|c|}{ Model 3‡ } \\
\hline & Mean & $95 \% \mathrm{Cl}$ & Mean & $95 \% \mathrm{Cl}$ & Mean & $95 \% \mathrm{Cl}$ \\
\hline \multicolumn{7}{|l|}{ Femoral neck } \\
\hline No use & 0.834 & $0.812,0.856$ & 0.835 & $0.814,0.857$ & 0.835 & $0.813,0.856$ \\
\hline$<1$ drinks/week & $0 \cdot 860$ & $0.836,0.884$ & $0 \cdot 870 \S$ & $0.847,0.892$ & $0.869 \S$ & $0.846,0.891$ \\
\hline 1-3 drinks/week & $0.897 \S$ & $0.864,0.929$ & $0.889 \S$ & $0.860,0.919$ & $0.891 \S$ & $0.861,0.921$ \\
\hline$>3$ drinks/week & $0.913 \S$ & $0.858,0.968$ & $0.897 \S$ & $0.848,0.946$ & $0.899 \S$ & $0.850,0.948$ \\
\hline \multicolumn{7}{|l|}{ Lumbar spine } \\
\hline No use & $1 \cdot 071$ & $1 \cdot 037,1 \cdot 105$ & 1.076 & $1 \cdot 043,1 \cdot 110$ & $1 \cdot 077$ & $1 \cdot 044,1 \cdot 110$ \\
\hline$<1$ drinks/week & $1 \cdot 068$ & $1 \cdot 030,1 \cdot 106$ & $1 \cdot 074$ & $1 \cdot 037,1 \cdot 110$ & $1 \cdot 073$ & $1 \cdot 036,1 \cdot 109$ \\
\hline 1-3 drinks/week & $1 \cdot 153 \S$ & $1 \cdot 103,1 \cdot 203$ & $1 \cdot 145 \S$ & $1 \cdot 099,1 \cdot 192$ & $1 \cdot 146 \$$ & $1 \cdot 100,1 \cdot 192$ \\
\hline$>3$ drinks/week & $1 \cdot 164 \S$ & $1 \cdot 082,1 \cdot 245$ & $1 \cdot 143$ & $1 \cdot 069,1 \cdot 217$ & $1 \cdot 139$ & $1 \cdot 065,1 \cdot 212$ \\
\hline
\end{tabular}

HT, hormone therapy; BMD, bone mineral density.

${ }^{*}$ Adjusted for study group (intervention $v$. control).

tAdjusted for age, BMI, HT use, duration of HT use, current smoking, use of Ca and vitamin D supplements, restricted mobility, disease or medication decreasing BMD, study group (intervention $v$. control).

$\ddagger$ Adjusted for age, BMI, HT use, duration of HT use, current smoking, use of Ca and vitamin D supplements, restricted mobility, disease or medication decreasing BMD, study group (intervention $v$. control), energy intake, dietary protein, dietary Ca, dietary vitamin $\mathrm{D}$, dietary $\mathrm{K}$.

$\S$ The difference between category no use $v$. other categories is statistically significant at the 0.05 level.

occur in alcohol-drinking elderly women ${ }^{(12,14,16,18,20,21,25)}$. However, in our sample there were no statistically significant differences in BMI across the categories of alcohol intake. There seemed to be an association between HT use and alcohol consumption in our study. A total of $73.7 \%$ women in the highest category of alcohol intake currently used or had used HT for some time during their life.

Mobility is another factor with positive impact on BMD. All elderly women consuming $>3$ drinks/week had normal mobility, whereas $9.6 \%$ of the abstaining women had restricted mobility. Higher physical activity among alcohol-consuming women was reported by Ganry et $a l^{(17)}$ and Mukamal et al. ${ }^{(21)}$. Alcohol-drinking women participating in the study conducted by Felson et al. ${ }^{(14)}$ were exercising less than non-drinking women. Based on the findings from this and previous studies, elderly women who drink alcohol seem to have a certain lifestyle pattern. They are more likely to smoke and exercise, have lower BMI and are more likely to use HT than alcoholabstaining elderly women.

The present study sought to investigate the impact of alcohol consumption on BMD at the femoral neck and lumbar spine in a homogeneous study population. There was repeated longitudinal information on alcohol consumption using the lifestyle questionnaire and BMD measurements, and the analytical approach selected allowed the correlation between repeated BMD measurements on the same participants to be taken into account. 
Strengths of the study also include the ability to control for well-established confounders, including lifestyle factors and dietary factors. However, limitations of our study are also recognized. Although data on alcohol consumption using the lifestyle questionnaire were collected from baseline and 3-year follow-up, the FFQ was distributed only at the follow-up examination. One of the drawbacks was also the relatively small sample size. Since data on dietary intake were available from only about half the women initially enrolled in the trial, we cannot rule out the possibility of attrition bias. However, the women participating in the study showed no differences in terms of main characteristics (age, BMI, HT use, duration of HT use and smoking) compared with those who did not participate. The proportions of women with restricted mobility $(9 \cdot 3 \%)$, non-prescribed use of $\mathrm{Ca}$ and vitamin D supplements (18.5\%) and disease or medication decreasing BMD (41.6\%) were higher in non-participating women. The study was part of a nonplacebo-controlled, parallel-group trial, where half of the sample received vitamin D and Ca supplementation. To rule out the possibility of an independent effect between alcohol intake and the $\mathrm{Ca}$ and vitamin $\mathrm{D}$ intervention (study group) on BMD, its interaction was tested prior to analysis and showed no significance. Additionally, the study group was added as a covariate to all models of analysis.

Ideally, the effect of alcohol consumption on BMD needs to be studied over a long period of time. The follow-up period of our study was 3 years, and therefore cannot fully address this issue. Another limitation was the fact that physical activity was not directly measured. However we did assess mobility, which reflects the ability to perform physical exercise. Also, the possibility of other unmeasured confounding factors cannot be ruled out.

\section{Conclusion}

The results from the OSTPRE-FPS cohort suggest that low alcohol intake may exert protective effects on bone health in elderly women. Further studies are needed to confirm these findings.

\section{Acknowledgements}

The OSTPRE-FPS was supported by the Finnish Cultural Foundation (Hulda Tossavainen Foundation), the Sigrid Juselius Foundation, the Academy of Finland and a Kuopio University Hospital EVO grant. The authors declare that they have no conflicts of interest. I.S. conducted data analysis, data interpretation and the writing of the first manuscript draft. A.T.E. and R.J. were responsible for the FFQ data and contributed to data interpretation. J.M. contributed to the data interpretation. J.S. performed BMD measurements. H.K., J.S.J. and M.T.T. were responsible for the original OSTPRE-FPS study design. All of the authors contributed to revising the manuscript and approved the final version. The authors thank Ms Seija Oinonen for technical support.

\section{References}

1. Johnell O, Kanis JA, Oden A et al. (2005) Predictive value of BMD for hip and other fractures. $J$ Bone Miner Res 20, 1185-1194.

2. Cauley JA, Hochberg MC, Lui LY et al. (2007) Long-term risk of incident vertebral fractures. JAMA 19, 2761-2767.

3. Kröger H, Tuppurainen M, Honkanen R et al. (1994) Bone mineral density and risk factors for osteoporosis a population-based study of 1600 perimenopausal women. Calcif Tissue Int 55, 1-7.

4. Saville PD (1965) Changes in bone mass with age and alcoholism. J Bone Joint Surg 47-A, 492-499.

5. Peris P, Parés A, Guañabenes N et al. (1992) Reduced spinal and femoral bone mass and deranged bone mineral metabolism in chronic alcoholics. Alcohol Alcohol 27, 619-625.

6. Sampson HW (1998) Alcohol's harmful effects on bone. Alcohol Health Res World 22, 190-194.

7. Klein RF (1997) Alcohol-induced bone disease: impact of ethanol on osteoblast proliferation. Alcohol Clin Exp Res 21, 392-399.

8. Sampson HW (1997) Alcohol, osteoporosis, and bone regulating hormones. Alcohol Clin Exp Res 21, 400-403.

9. Turner RT (2000) Skeletal response to alcohol. Alcohol Clin Exp Res 24, 1693-1701.

10. Angus RM, Sambrook PN, Pocock NA et al. (1998) Dietary intake and bone mineral density. Bone Miner 4, 265-277.

11. Laitinen K, Välimäki M \& Keto P (1991) Bone mineral density measured by dual-energy X-ray absorptiometry in healthy Finnish women. Calcif Tissue Int 48, 224-231.

12. Holbrook TL \& Barrett-Connor E (1993) A prospective study of alcohol consumption and bone mineral density. BMJ 306, 1506-1509.

13. Nguyen TV, Kelly PJ, Sambrook PN et al. (1994) Lifestyle factors and bone density in the elderly: implications for osteoporosis prevention. J Bone Miner Res 9, 1339-1346.

14. Felson DT, Zhang Y, Hannan MT et al. (1995) Alcohol intake and bone mineral density in elderly men and women. Am J Epidemiol 142, 485-492.

15. Orwoll ES, Bauer DC, Vogt TM et al. (1996) Axial bone mass in older women. Ann Intern Med 124, 187-196.

16. Feskanich D, Willet W, Stampfer M et al. (1996) Protein consumption and bone fractures in women. $\mathrm{Am} \mathrm{J}$ Epidemiol 143, 472-479.

17. Ganry O, Baudoin C \& Fardellone P (2000) Effect of alcohol intake on bone mineral density in elderly women. Am J Epidemiol 151, 773-780.

18. Rapuri PB, Gallagher JC, Balhorn KE et al. (2000) Alcohol intake and bone metabolism in elderly women. Am J Clin Nutr 72, 1206-1213.

19. Williams FMK, Cherkas LF, Spector TD et al. (2005) The effect of moderate alcohol consumption on bone mineral density: a study of female twins. Ann Rheum Dis 64, 309-310.

20. Wosje KS \& Kalkwarf HJ (2007) Bone density in relation to alcohol intake among men and women in the United States. Osteoporos Int 18, 391-400.

21. Mukamal KJ, Robbins JA, Cauley JA et al. (2007) Alcohol consumption, bone density, and hip fracture among older adults: the Cardiovascular Health Study. Osteoporos Int 18, 593-602.

22. Pedrera-Zamorano JD, Lavado-Garcia JM, Roncero-Martin $\mathrm{R}$ et al. (2009) Effect of beer drinking on ultrasound bone mass in women. Nutrition 25, 1057-1063. 
23. Tucker KL, Jugdaohsingh R, Powell JJ et al. (2009) Effects of beer, wine, and liquor intakes on bone mineral density in older men and women. Am J Clin Nutr 89, 1188-1196.

24. Stevenson JC, Lees B, Devenport M et al. (1989) Determinants of bone density in normal women: risk factors for future osteoporosis? BMJ 298, 924-928.

25. Hansen MA, Overgaard K, Riis BJ et al. (1991) Potential risk factors for development of postmenopausal osteoporosis examined over a 12-year period. Osteoporos Int 1, 95-102.

26. Bauer DC, Browner WS, Cauley JA et al. (1993) Factors associated with appendicular bone mass in older women. Ann Intern Med 118, 657-665.

27. MacInnis RJ, Cassar C, Nowson CA et al. (2003) Determinants of bone density in 30- to 65-year-old women: a co-twin study. J Bone Miner Res 18, 1650-1656.

28. Tivis LJ \& Tivis RD (2008) One-per-occasion or less: are moderate-drinking postmenopausal women really healthier than their nondrinking and heavier-drinking peers? Alcohol Clin Exp Res 32, 1670-1680.

29. Grainge MJ, Coupland CAC, Cliffe SJ et al. (1998) Cigarette smoking, alcohol and caffeine consumption, and bone mineral density in postmenopausal women. Osteoporos Int 8, 355-363.

30. Klatsky AL (2007) Alcohol, cardiovascular diseases and diabetes mellitus. Pharmacol Res 55, 237-247.

31. Onland-Moret NC, Peeters PHM, van der Schouw YT et al. (2005) Alcohol and endogenous sex steroid levels in postmenopausal women: a cross-sectional study. I Clin Endocrinol Metab 90, 1414-1419.

32. Gavaler JS (2005) Should we consider an acceptable drinking level specifically for postmenopausal women? Preliminary findings from the postmenopausal health disparities study. Alcohol Alcohol 40, 469-473.

33. Kärkkäinen M, Tuppurainen M, Salovaara K et al. (2010) Effect of calcium and vitamin D supplementation on bone mineral density in women aged 65-71 years: a randomized population-based trial (OSTPRE-FPS). Osteoporos Int 21, $2047-2055$.

34. Honkanen R, Alhava EM, Saarikoski S et al. (1991) Osteoporosis risk factors in perimenopausal women. Calcif Tissue Int 49, Suppl., S74-S75.

35. Erkkilä AT, Järvinen R, Karvonen H et al. (2012) Validation of a semi-quantitative food frequency questionnaire using food records as reference in older women in the Kuopio Fracture Prevention Study (OSTPRE-FPS). Public Health Nutr 15, 635-639.
36. Kärkkäinen M, Rikkonen T, Kröger H et al. (2009) Physical tests for patient selection for bone mineral density measurements in postmenopausal women. Bone $\mathbf{4 4}$, 660-665.

37. Darling A, Millward D, Torgerson D et al. (2009) Dietary protein and bone health; a systematic review and meta-analysis. Am J Clin Nutr 90, 1674-1692.

38. Tang BMP, Eslick GD, Nowson C et al. (2007) Use of calcium or calcium in combination with vitamin D supplementation to prevent fractures and bone loss in people aged 50 years and older. Lancet 370, 657-666.

39. Cranney A, Weiler HA, O'Donnell S et al. (2008) Summary of evidence-based review on vitamin D efficacy and safety in relation to bone health. Am J Clin Nutr 88, issue 2, 513S-519S.

40. Lanham-New SA (2008) The balance of bone health: tipping the scales in favor of potassium-rich, bicarbonate-rich foods. J Nutr 138, issue 1, 172S-177S.

41. Zhu K, Devine A \& Prentice R (2009) The effects of high potassium consumption on bone mineral density in a prospective cohort study of elderly postmenopausal women. Osteoporos Int 20, 335-340.

42. Halme JK, Seppä K, Alho H et al. (2010) Alcohol consumption all-cause mortality among elderly in Finland. Drug Alcohol Depend 106, 212-218.

43. MacDonald HM (2009) Alcohol and recommendations for bone health; should we still exercise caution? $A m$ J Clin Nutr 89, 999-1000.

44. Aira M, Hartikainen S \& Sulkava R (2008) Drinking alcohol for medicinal purposes by people aged older 75: a community-based interview study. Fam Pract 25, 445-449.

45. Jones G, Nguyen T, Sambrook P et al. (1994) Progressive loss of bone in the femoral neck in elderly people: longitudinal findings from the Dubbo osteoporosis epidemiology study. BMJ 309, 691-695.

46. Greenspan SL, Maitland LA, Myers ER et al. (1994) Femoral bone loss progresses with age: a longitudinal study in women over age 65. J Bone Miner Res 9, 1959-1965.

47. Muraki S, Yamamoto S, Ishibashi H et al. (2004) Impact of degenerative spinal disease on bone mineral density of the lumbar spine in elderly women. Osteoporos Int 15, $724-728$

48. Ricci TA, Hdeymsfield SB, Pierson RN et al. (2001) Moderate energy restriction increases bone resorption in obese postmenopausal women. Am J Clin Nutr 73, 347-352. 